\title{
High-energy Neutrino Astronomy: From AMANDA to IceCube
}

\author{
Francis Halzen \\ Department of Physics, University of Wisconsin, Madison, WI 53706
}

\begin{abstract}
Kilometer-scale neutrino detectors such as IceCube are discovery instruments covering nuclear and particle physics, cosmology and astronomy. Examples of their multidisciplinary missions include the search for the particle nature of dark matter and for additional small dimensions of space. In the end, their conceptual design is very much anchored to the observational fact that Nature accelerates protons and photons to energies in excess of $10^{20}$ and $10^{13} \mathrm{eV}$, respectively. The cosmic ray connection sets the scale of cosmic neutrino fluxes. In this context, we discuss the first results of the completed AMANDA detector and the reach of its extension, IceCube.
\end{abstract}

\section{Neutrinos Associated with the Highest Energy Cosmic Rays}

The flux of cosmic rays is summarized in Fig. 1a,b (Gaisser 2002). The energy spectrum follows a broken power law. The two power laws are separated by a feature referred to as the "knee"; see Fig. 1a. There is evidence that cosmic rays, up to several $\mathrm{EeV}$, originate in galactic sources. This correlation disappears in the vicinity of a second feature in the spectrum dubbed the "ankle". Above the ankle, the gyroradius of a proton exceeds the size of the galaxy and it is generally assumed that we are witnessing the onset of an extragalactic component in the spectrum that extends to energies beyond $100 \mathrm{EeV}$. Experiments indicate that the highest energy cosmic rays are predominantly protons. Above a threshold of $50 \mathrm{EeV}$ these protons interact with CMBR photons and therefore lose their energy to pions before reaching our detectors. This limits their sources to tens of $\mathrm{Mpc}$, the so-called Greissen-Zatsepin-Kuzmin cutoff.

Models for the origin of the highest energy cosmic rays fall into two categories, top-down and bottom-up. In top-down models it is assumed that the cosmic rays are the decay products of cosmological remnants with Grand Unified energy scale $M_{G U T} \sim 10^{24} \mathrm{eV}$. These models predict neutrino fluxes most likely within reach of AMANDA, and certainly IceCube.

In bottom-up scenarios it is assumed that cosmic rays originate in cosmic accelerators. Accelerating particles to $\mathrm{TeV}$ energy and above requires massive bulk flows of relativistic charged particles. These are likely to originate from the exceptional gravitational forces in the vicinity of black holes. Examples include the dense cores of exploding stars, inflows onto super-massive black holes at the centers of active galaxies and annihilating black holes or neutron stars. Before leaving the source, accelerated particles pass through intense radiation fields or dense clouds of gas surrounding the black hole. This results in interactions 

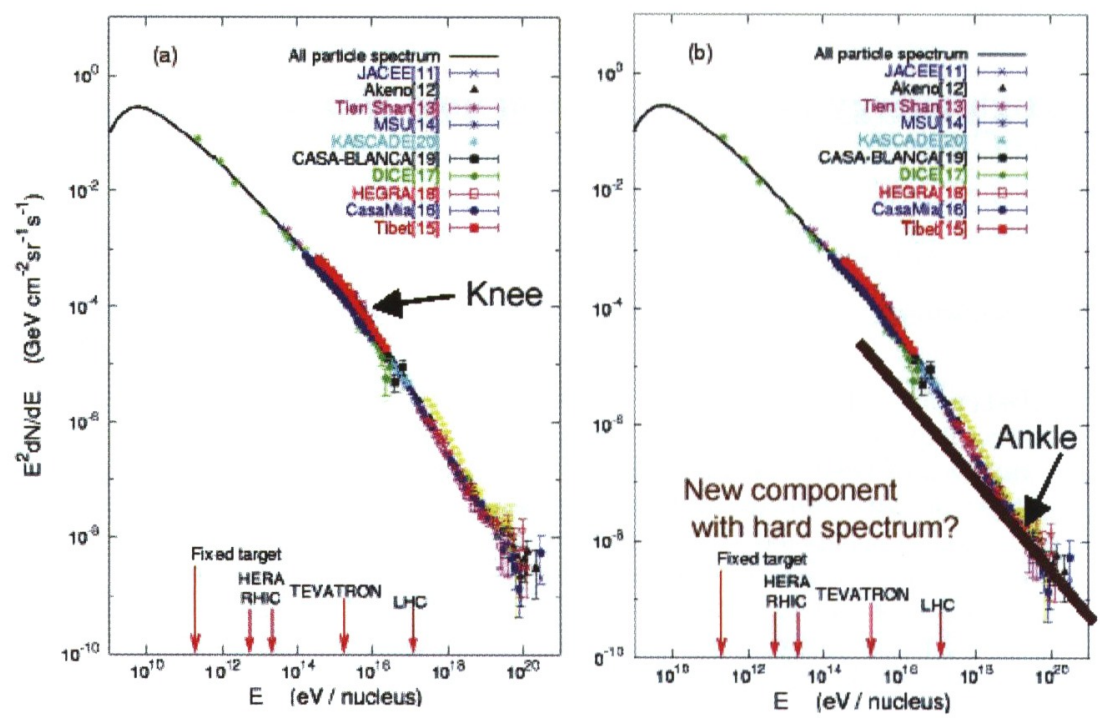

Figure 1. At the energies of interest here, the cosmic ray spectrum consists of a sequence of 3 power laws. The first two are separated by the "knee" (left panel), the second and third by the "ankle". There is evidence that the cosmic rays beyond the ankle are a new population of particles produced in extragalactic sources; see right panel.

producing pions decaying into secondary photons and neutrinos that accompany the primary cosmic ray beam as illustrated in Fig. 2.

How many neutrinos are produced in association with the cosmic ray beam? The answer to this question, among many others (Gaisser, Halzen \& Stanev $1995)$, provides the rationale for building kilometer-scale neutrino detectors. We first consider a neutrino beam produced at an accelerator laboratory; see Fig. 2. Here the target absorbs all parent protons as well as the muons, electrons and gamma rays produced. A pure neutrino beam exits the dump. If nature constructed such a "hidden source" in the heavens, conventional astronomy will not reveal it. It cannot be the source of the cosmic rays, however, because the dump would have to be partially transparent to protons. The extreme opposite case is a "transparent source" where the accelerated proton interacts once and escapes the dump after producing photons as well as neutrinos. Elementary particle physics is now sufficient to relate all particle fluxes because a fraction ( $1 / 6$ to $1 / 2$ depending on the energy) of the interacting proton goes into pion production. This energy is equally shared between gamma rays and neutrinos, of which one half are muon-neutrinos. Therefore, at most one quarter of the energy ends up in muon-neutrinos compared to cosmic rays. The flux of a transparent cosmic ray source is often referred to as the Waxman-Bahcall flux (Bahcall \& Waxman 2001). It is easy to derive and the derivation is revealing.

Fig. 1b shows a fit to the observed cosmic ray spectrum assuming an extragalactic component fitted above the "ankle". The energy content of this component is $\sim 3 \times 10^{-19} \mathrm{erg} \mathrm{cm}^{-3}$, assuming an $E^{-2}$ energy spectrum with a 


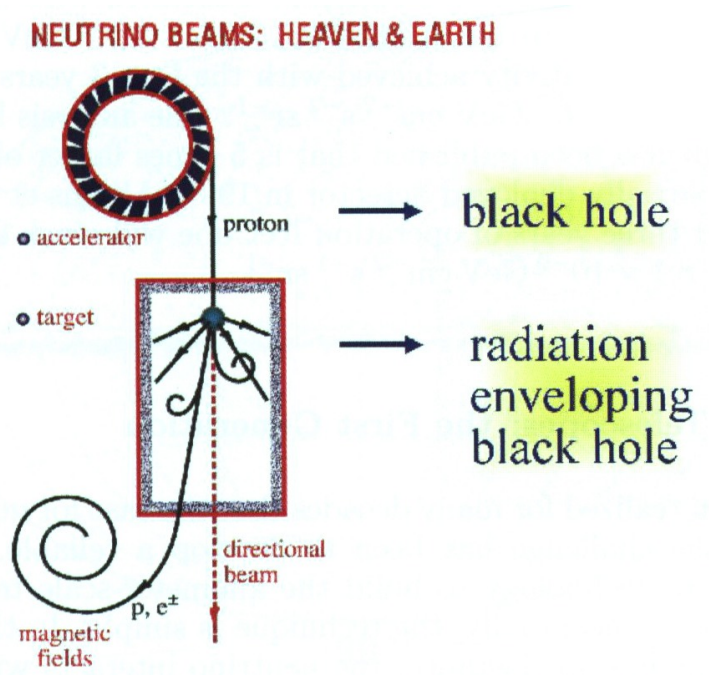

Figure 2. Diagram of cosmic ray accelerator producing photons and neutrinos.

GZK cutoff. The power required to generate this energy density in the Hubble time of $10^{10}$ years is $\sim 3 \times 10^{37} \mathrm{erg} \mathrm{s}^{-1}$ per $(\mathrm{Mpc})^{3}$. This works out to (Gaisser 1997)

- $\sim 3 \times 10^{39} \mathrm{erg} \mathrm{s}^{-1}$ per galaxy,

- $\sim 3 \times 10^{42} \mathrm{erg} \mathrm{s}^{-1}$ per cluster of galaxies,

- $\sim 2 \times 10^{44} \mathrm{erg} \mathrm{s}^{-1}$ per active galaxy, or

- $\sim 2 \times 10^{52}$ erg per cosmological gamma ray burst.

The coincidence between these numbers and the observed electromagnetic energy output of these sources explains why they have emerged as the leading candidates for the cosmic ray accelerators. The coincidence is consistent with the relationship between cosmic rays and photons built into the "transparent" source previously introduced. The relationship can be extended to neutrinos.

Assuming the same energy density of $\rho_{E} \sim 3 \times 10^{-19} \mathrm{erg} \mathrm{cm}^{-3}$ in neutrinos with a spectrum $E_{\nu} d N / d E_{\nu} \sim E^{-\gamma} \mathrm{cm}^{-2} \mathrm{~s}^{-1} \mathrm{sr}^{-1}$ that continues up to a maximum energy $E_{m a x}$, the neutrino flux follows from $\int E_{\nu} d N / d E_{\nu}=c \rho_{E} / 4 \pi$. For $\gamma=1$ and $E_{\max }=10^{8} \mathrm{GeV}$, the generic source of the highest energy cosmic rays produces 50 detected muon neutrinos per $\mathrm{km}^{2}$ per year (Gaisser 1997). [Here we have folded the predicted flux with the probability that the neutrino is actually detected given by (Gaisser et al. 1995) the ratio of the muon and neutrino interaction lengths in ice, $\lambda_{\mu} / \lambda_{\nu}$.] The number depends weakly on $E_{\max }$ and $\gamma$. A similar analysis can be performed for galactic sources (Gaisser et al. 1995).

As previously stated, for one interaction and only one, the neutrino flux should be reduced by a factor $\sim 4$. On the other hand, there are more cosmic rays in the universe producing neutrinos than observed at earth because of the 
GZK-effect. The diffuse muon neutrino flux associated with the highest energy cosmic rays is estimated to be $E_{\nu}{ }^{2} d N / d E_{\nu} \sim 5 \times 10^{-8} \mathrm{GeV} \mathrm{cm}^{-2} \mathrm{~s}^{-1} \mathrm{sr}^{-1}$, to be compared to the sensitivity achieved with the first 3 years of the completed AMANDA detector of $10^{-7} \mathrm{GeV} \mathrm{cm}{ }^{-2} \mathrm{~s}^{-1} \mathrm{sr}^{-1}$. The analysis has not been completed but a limit has been published that is 5 times larger obtained with data taken with the partially deployed detector in 1997 (Ahrens et al. 2003). On the other hand, after three years of operation IceCube will reach a diffuse flux limit of $E_{\nu}^{2} d N / d E_{\nu}=8.1 \times 10^{-9} \mathrm{GeV} \mathrm{cm}^{-2} \mathrm{~s}^{-1} \mathrm{sr}^{-1}$.

\section{Neutrino Telescopes: the First Generation}

While it has been realized for many decades that the case for neutrino astronomy is compelling, the challenge has been to develop a reliable, expandable and affordable detector technology to build the kilometer-scale telescopes required to do the science. Conceptually, the technique is simple. In the case of a highenergy muon neutrino, for instance, the neutrino interacts with a hydrogen or oxygen nucleus in deep ocean water and produces a muon traveling in nearly the same direction as the neutrino. The blue Cerenkov light emitted along the muon's kilometer-long trajectory is detected by strings of photomultiplier tubes deployed at depth shielded from radiation. The orientation of the Cerenkov cone reveals the neutrino direction.

The AMANDA detector, using natural 1 mile deep Antarctic ice as a Cerenkov detector, has operated for more than 3 years in its final configuration of 680 optical modules on 19 strings. The detector is in steady operation collecting roughly four neutrinos per day using fast on-line analysis software. Its performance has been calibrated by reconstructing muons produced by atmospheric muon neutrinos (Andres et al. 2001).

Using the first of 3 years of AMANDA II data, the AMANDA collaboration is performing a (blind) search for the emission of muon neutrinos from spatially localized directions in the northern sky (Karle et al. 2002). Only the year 2000 data have been unblinded. The sky-plot is shown in Fig. 3. 90\% upper limits on the fluency of point sources is at the level of $2 \times 10^{-7} \mathrm{GeV} \mathrm{cm}^{-2} \mathrm{~s}^{-1}$ or $3 \times 10^{-10} \mathrm{erg} \mathrm{cm}^{-2} \mathrm{~s}^{-1}$, averaged over declination. This corresponds to a flux of $2 \times 10^{-8} \mathrm{~cm}^{-2} \mathrm{~s}^{-1}$ integrated above $10 \mathrm{GeV}$. The most significant excess is 8 events observed on an expected background of 2.1, occurring at approximately $68 \mathrm{deg} \mathrm{N}$ dec, $21.1 \mathrm{hr}$ R.A; for details see Hauschildt \& Steele (2003). Unblinding the data collected in 2001, 2002 may reveal sources or confirm the consistency of the year 2000 sky-plot with statistical fluctuations on the atmospheric neutrino background.

With this search the AMANDA II detector has reached a high-energy effective telescope area of $25,000 \sim 40,000 \mathrm{~m}^{2}$, depending on declination. This represents an interesting milestone: sources with an $E^{-2}$ spectrum should be observed provided the number of gamma rays and neutrinos are roughly equal as expected from cosmic ray accelerators producing pions (Alvarez-Muniz \& Halzen 2002).

Overall, AMANDA represents a proof of concept for the kilometer-scale neutrino observatory, IceCube (Wissing 2003), now under construction. The detector is described elsewhere in this volume (Halzen 2003). 




Figure 3. Skymap showing declination and right ascension of neutrinos detected by the completed AMANDA II detector during its first Antarctic winter of operation in 2000 .

\section{References}

Ahrens, J. et al. (AMANDA Collaboration) 2003, Phys. Rev. Lett. 90, 251101, astro$\mathrm{ph} / 0303218$

Alvarez-Muniz, J. \& Halzen, F. 2002, Ap. J. 576, L33; Crocker, R. M. 2002, PhD thesis (University of Melbourne)

Andres, E. et al. (AMANDA Collaboration) 2001, Nature 410, 441; 2000, Astroparticle Physics 13, 1

Bahcall, J. N. \& Waxman, E. 2001, Phys. Rev. D64, 023002

Gaisser, T. K. 2002, in Proceedings of the 31st International Conference on High Energy Physics, Amsterdam, The Netherlands, July 2002

Gaisser, T. K. 1997, astro-ph/9707283, talk presented at the OECD Megascience Forum, Taormina, Italy, 1997

Gaisser, T. K., Halzen, F. \& Stanev, T. 1995, Phys. Rept. 258, 173 [Erratum 271, 355 (1995)], hep-ph/9410384

Halzen, F. 2003, these proceedings

Hauschildt, T. \& Steele, D. 2003, in Proc. of the 28th International Cosmic Ray Conference, Tsukuba, Japan, August 2003

Karle, A. et al. (IceCube Collaboration) 2002, in Proc. of the XXth International Conference on Neutrino Physics and Astrophysics, Munich, Germany, May 2002, astro-ph/020955

Wissing, H. 2003, PhD thesis (Humbold University, Berlin); Particle Astrophysics (in press) and http://icecube.wisc.edu/science/sci-tech-docs/ 